\title{
On Cui-Kano's Characterization Problem on Graph Factors
}

\author{
H. L. Lu ${ }^{1}$ and David G. L. Wang ${ }^{2}$ \\ ${ }^{1}$ Department of Mathematics \\ Xi'an Jiaotong University, Xi'an 710049, P. R. China \\ ${ }^{1}$ luhongliang215@sina.com \\ ${ }^{2}$ Beijing International Center for Mathematical Research \\ Peking University, Beijing 100871, P. R. China \\ ${ }^{2}$ wgl@math.pku.edu.cn
}

\begin{abstract}
An $H_{n}$-factor of a graph $G$ is defined to be a spanning subgraph $F$ of $G$ such that each vertex has degree belonging to the set $\{1,3,5, \ldots, 2 n-1,2 n\}$ in $F$. In this paper, we investigate $H_{n}$-factors of graphs by using Lovász's structural descriptions to the degree prescribed subgraph problem. We find some sufficient conditions for the existence of an $H_{n}$-factor of a graph. In particular, we make progress on the characterization problem for a special family of graphs proposed by Cui and Kano in 1988.
\end{abstract}

Keywords: Lovász's structural description, $H_{n}$-factor, $H_{n}$-decomposition 2010 AMS Classification: 05C75

\section{Introduction}

Let $G$ be a simple graph with vertex set $V(G)$. Let $f, g: V(G) \rightarrow \mathbb{Z}$ be functions on $V(G)$. A $(g, f)$-factor of $G$ is a spanning subgraph $F$ such that

$$
g(v) \leq d_{F}(v) \leq f(v)
$$

for any vertex $v$, where $d_{F}(v)$ is the degree of $v$ in $F$. In particular, if there exist integers $a$ and $b$ such that $g(v)=a$ and $f(v)=b$ for all vertices $v$, then the $(g, f)$ factor is called an $[a, b]$-factor. For example, connected [2,2]-factors are nothing but Hamiltonian cycles, and [1,1]-factors are perfect matchings. There is a large amount of literature on graph factors, see Plummer [10], Liu and $\mathrm{Yu}$ [12], and Akiyama and Kano [1] for surveys. For connected factors, we refer the reader to Kouider and Vestergaard [6].

Let $H$ be a function associating a subset of $\mathbb{Z}$ with each vertex of $G$, called a degree prescription. It is natural to generalize $(g, f)$-factors to $H$-factors, i.e., spanning subgraphs $F$ such that

$$
d_{F}(v) \in H(v)
$$


for all vertices $v$. Let $F$ be a spanning subgraph of $G$. Following Lovász [9], one may measure the "deviation" of $F$ from the condition (1.1) by

$$
\delta_{H}(F)=\sum_{v \in V(G)} \min \left\{\left|d_{F}(v)-h\right|: h \in H(v)\right\} .
$$

Moreover, the "solvability" of (1.1) can be characterized by

$$
\delta(H)=\min \left\{\delta_{H}(F): F \text { is a spanning subgraph of } G\right\} .
$$

The subgraph $F$ is said to be $H$-optimal if $\delta_{H}(F)=\delta(H)$. It is clear that $F$ is an $H$-factor if and only if $\delta_{H}(F)=0$, and any $H$-factor (if exists) is $H$-optimal.

In [8], Lovász proposed the problem of determining the value of $\delta(H)$, called the degree prescribed subgraph problem. Let

$$
H=\left\{h_{1}, h_{2}, \ldots, h_{n}\right\}
$$

be a set of integers, where $h_{1}<h_{2}<\cdots<h_{n}$. It is said to be an allowed set if each of its gaps has at most one integer, i.e.,

$$
h_{i+1}-h_{i} \leq 2, \quad \forall 1 \leq i \leq n-1 \text {. }
$$

We say that a prescription $H$ is allowed if $H(v)$ is an allowed set for all vertices $v$. Lovász [9] built up a whole theory to the degree prescribed subgraph problem in case that $H$ is an allowed prescription. He showed that the problem is NP-complete without the restriction that $H$ is allowed. Cornuéjols [3] provided the first polynomial algorithm for the problem with $H$ allowed.

A special case of the degree prescribed subgraph problem is the so-called $f$-parity subgraph problem, i.e., the problem with

$$
H(v)=\{\ldots, f(v)-4, f(v)-2, f(v)\}
$$

for some function $f: V(G) \rightarrow \mathbb{Z}$. The first investigation of the $f$-parity subgraph problem is due to Amahashi [2], who gave a Tutte type characterization for graphs having a global odd factor. Let $S$ be a subset of $V(G)$. Denote by $G-S$ the subgraph of $G$ obtained by removing all vertices in $S$. Denote by $o(G)$ the number of odd components of $G$. Let $n \geq 2$ be an integer independent of the number of vertices of $G$. Let $H_{o}$ be the prescription associating the first $n$ positive odd integers with each vertex, i.e.,

$$
H_{o}(v)=\{1,3,5, \ldots, 2 n-1\}
$$

Theorem 1.1 (Amahashi) A graph $G$ has an $H_{o}$-factor if and only if

$$
o(G-S) \leq(2 n-1)|S|, \quad \forall S \subseteq V(G) .
$$


For general odd value functions $f$, Cui and Kano [4] established a Tutte type theorem. Noticing the form of the condition (1.3), they asked the question of characterizing graphs $G$ in terms of graph factors such that

$$
o(G-S) \leq 2 n|S|, \quad \forall S \subseteq V(G)
$$

For more studies on the $f$-parity subgraph problem, see Topp and Vestergaard [11], and Kano, Katona, and Szabó [5].

Motivating by solving Cui-Kano's problem, we consider the degree prescribed subgraph problem for the special prescription

$$
H_{n}(v)=H_{o}(v) \cup\{2 n\}=\{1,3,5, \ldots, 2 n-1,2 n\} .
$$

We shall study the structure of graphs which have no $H_{n}$-factors by using Lovász's theory [9]. Consequently, we obtain that any graph satisfying the condition (1.4) contains an $H_{n}$-factor.

Besides many applications of Lovász's structural description for special families of graphs and special allowed prescriptions, there is much attention paid to finding sufficient conditions for the existence of an $H$-factor in a graph for special prescriptions $H$, see [10]. In this paper, we also give some sufficient conditions for the existence of an $H_{n}$-factor in a graph.

\section{The main result}

In this section, we study $H_{n}$-factors of graphs based on Lovász's structural description of the degree prescribed subgraph problem.

Let $H$ be an allowed prescription. Denote by $I_{H}(v)$ the set of vertex degrees in all $H$-optimal subgraphs, i.e.,

$$
I_{H}(v)=\left\{d_{F}(v) \mid F \text { is } H \text {-optimal }\right\} .
$$

Comparing the set $I_{H}(v)$ with $H(v)$, one may partition the vertex set $V(G)$ into four classes:

$$
\begin{aligned}
& C_{H}=\left\{v \in V(G): I_{H}(v) \subseteq H(v)\right\}, \\
& A_{H}=\left\{v \in V(G) \backslash C_{H}: \min I_{H}(v) \geq \max H(v)\right\}, \\
& B_{H}=\left\{v \in V(G) \backslash C_{H}: \max I_{H}(v) \leq \min H(v)\right\}, \\
& D_{H}=V(G) \backslash A_{H} \backslash B_{H} \backslash C_{H} .
\end{aligned}
$$

It is clear that the 4-tuple $\left(A_{H}, B_{H}, C_{H}, D_{H}\right)$ is a pairwise disjoint partition of $V(G)$. We call it the $H$-decomposition of $G$. In fact, the four subsets can be distinguished 
according to the contributions of their members to the deviation (1.2). A graph $G$ is said to be $H$-critical if it is connected and $D_{H}=V(G)$.

In [9, Theorem (2.1)], Lovász gave the following property for the subset $D_{H}$.

Lemma 2.1 (Lovász) If $D_{H} \neq \emptyset$, then the intersection

$$
\left[\min I_{H}(v), \max I_{H}(v)\right] \cap H(v)
$$

contains no consecutive integers for any vertex $v \in D_{H}$.

In [9, Corollary (2.4)], Lovász gave the following structural result.

Lemma 2.2 (Lovász) There is no edge between $C_{H}$ and $D_{H}$.

For any subset $S \subseteq V(G)$, denote by $G[S]$ the subgraph induced by $S$. Denote the number of components of $G[S]$ by $c(S)$, and the number of odd components of $G[S]$ by $o(S)$. In [9, Theorem (4.3)], Lovász established the formula

$$
\delta(H)=c\left(D_{H}\right)+\sum_{v \in B_{H}} \min H(v)-\sum_{v \in A_{H}} \max H(v)-\sum_{v \in B_{H}} d_{G-A_{H}}(v) .
$$

By definition, $G$ contains no $H$-factors if and only if $\delta(H)>0$. This yields the next lemma immediately.

Lemma 2.3 (Lovász) A graph $G$ contains no $H$-factors if and only if

$$
c\left(D_{H}\right)+\sum_{v \in B_{H}} \min H(v)>\sum_{v \in A_{H}} \max H(v)+\sum_{v \in B_{H}} d_{G-A_{H}}(v) .
$$

Let $X \subseteq V(G)$. For any vertex $v$, define

$$
H_{X}(v)=\{h-e(v, X): h \in H(v)\},
$$

where $e(v, X)$ is the number of edges from $v$ to $X$. In [9, Theorem (4.2)], Lovász showed that each component of $G\left[D_{H}\right]$ is $H^{\prime}$-critical where $H^{\prime}=H_{B_{H}}$. He [9, Lemma (4.1)] also obtained that $\delta(H)=1$ if $G$ is $H$-critical. This leads to the next lemma.

Lemma 2.4 (Lovász) If $D_{H} \neq \emptyset$, then for any component $T$ of the subgraph $G\left[D_{H}\right]$, and any $H$-optimal subgraph $F$ of $T$, we have $\delta_{H^{\prime}}(F)=1$.

This paper concerns $H_{n}$-decompositions where $H_{n}$ is defined by (1.5). For convenience, we often use another prescription $H_{n}^{*}$ defined by

$$
H_{n}^{*}(v)=H_{n}(v) \cup\{-1\}=\{-1,1,3,5, \ldots, 2 n-1,2 n\} .
$$

Here is the main result of this paper. 
Theorem 2.5 Let $G$ be a graph without odd components. If $G$ contains no $H_{n}$-factors, then there exists a nonempty subset $S \subset V(G)$ such that the subgraph $G-S$ contains at least $2 n|S|+1$ odd components, each of which contains no $H_{n}$-factors.

Proof. By the definition (2.3), the graph $G$ contains no $H_{n}^{*}$-factors. Let $(A, B, C, D)$ be the $H_{n}^{*}$-decomposition of $G$. We shall show that the subset $A$ can be taken as the required $S$.

Since $\min H_{n}^{*}=-1$, we have $B=\emptyset$, and the inequality (2.1) reduces to

$$
c(D)>2 n|A| .
$$

This implies $D \neq \emptyset$. Let $T$ be a component of the subgraph $G[D]$, and $F$ an $H_{n}^{*}$-optimal subgraph of $T$. Since $B=\emptyset$, we see that $H_{B}=H_{n}^{*}$. So $\delta_{H_{n}^{*}}(F)=1$ by Lemma 2.4. Therefore, there exists a vertex, say $v_{0} \in V(T)$, such that

$$
\min \left\{\left|d_{F}(v)-h\right|: h \in H_{n}^{*}(v)\right\}= \begin{cases}1, & \text { if } v=v_{0} ; \\ 0, & \text { if } v \in V(T) \backslash\left\{v_{0}\right\} .\end{cases}
$$

On the other hand, assume that $\max I_{H_{n}^{*}}(v) \geq 2 n$ for some $v \in D$. By Lemma 2.1, we have

$$
\min I_{H_{n}^{*}}(v) \geq 2 n
$$

It follows immediately that $v \in A$, a contradiction. Thus $\max I_{H_{n}^{*}}(v) \leq 2 n-1$, namely

$$
d_{F}(v) \leq 2 n-1
$$

for any vertex $v \in T$. Consequently, the formula (2.5) implies that the degree $d_{F}\left(v_{0}\right)$ is even, while $d_{F}(v)$ is odd for any $v \in V(T) \backslash\left\{v_{0}\right\}$. Since the sum $\sum_{v \in V(T)} d_{F}(v)$ is even, we deduce that $T$ is an odd component of $G[D]$.

Assume that $A=\emptyset$. Since $B=\emptyset$, by Lemma 2.2, we see that $T$ is an odd component of $G$. But $G$ has no odd components, a contradiction. So $A \neq \emptyset$.

By Lemma 2.2 and the inequality (2.4), we have

$$
2 n|A|<c(D)=o(D) \leq o(C)+o(D)=o(C \cup D)=o(G-A) .
$$

Namely, the subgraph $G-A$ has at least $2 n|A|+1$ odd components. In view of (2.5), any component $T$ of $G[D]$ has no $H_{n}^{*}$-factors. Hence $T$ has no $H_{n}$-factors. This completes the proof.

For graphs satisfying Cui-Kano's condition (1.4), we obtain the following corollary immediately.

Corollary 2.6 Any graph $G$ satisfying the condition (1.4) contains an $H_{n}$-factor. 
Write $g=|V(G)|$. Noting that the condition " $G$ has no odd components" implies that $g$ is even. Considering graphs $G$ with $g$ odd in contrast, we have the following result.

Theorem 2.7 Let $G$ be a connected graph with $g$ odd. Suppose that

$$
o(G-S) \leq 2 n|S|, \quad \forall S \subseteq V(G), S \neq \emptyset .
$$

Then either $G$ contains an $H_{n}$-factor, or $G$ is $H_{n}^{*}$-critical.

Proof. Suppose that $G$ contains no $H_{n}$-factors. Let

$$
(A, B, C, D)
$$

be the $H_{n}^{*}$-decomposition of $G$. From the proof of Theorem 2.5, we see that $B=\emptyset$, and obtain the inequality (2.6). Together with the condition (2.7), we see that $A=\emptyset$. Since $G$ is connected, we find that $C=\emptyset$ by Lemma 2.2. Hence $G$ is $H_{n}^{*}$-critical. This completes the proof.

We remark that the condition (1.4) is not necessary for the existence of an $H_{n^{-}}$ factor in a graph. Consider the graph

$$
G=K_{1}+(2 n+1) K_{2 n+1}
$$

obtained by linking a vertex $K_{1}$ to all vertices in $2 n+1$ copies of the complete graph $K_{2 n+1}$. Denote by $C_{j}(1 \leq j \leq 2 n+1)$ the $j$-th copy of $K_{2 n+1}$. Let $v_{j} \in V\left(C_{j}\right)$. Let $F$ be the factor consisting of the following $2 n+2$ components:

$$
C_{1}-v_{1}, C_{2}-v_{2}, \ldots, C_{2 n}-v_{2 n}, C_{2 n+1}, G\left[v_{0}, v_{1}, \ldots, v_{2 n}\right] .
$$

It is easy to verify that $F$ is an $H_{n}$-factor. However, taking the subset $S$ to be the single vertex $v_{0}$, we see that the condition (1.4) does not hold for $G$.

To end this section, we point out that the coefficient $2 n$ in the condition (1.4) is a sharp bound in the sense that for any $\epsilon>0$, there exists a graph $G$ with a subset $S \subseteq V(G)$ satisfying

$$
o(G-S)<(2 n+\epsilon)|S|,
$$

and that $G$ contains no $H_{n}$-factors. Recall that an $[a, b]$-factor is a factor $F$ such that $a \leq d_{F}(v) \leq b$ for all vertices $v$. We need Las Vergnas's theorem [7].

Theorem 2.8 (Las Vergnas) A graph $G$ contains a $[1, n]$-factor if and only if for all subsets $S \subseteq V(G)$, the number of isolated vertices in the subgraph $G-S$ is at most $n|S|$.

Theorem 2.9 For any $\epsilon>0$, there exists a graph $G$ with a subset $S \subseteq V(G)$ satisfying the $\epsilon$-condition (2.8) but with no $H_{n}$-factors. 
Proof. Let $m$ be an integer such that $m>1 / \epsilon$. Let $V_{m}$ be a set of $m$ isolated vertices, and $V_{2 n m+1}$ a set of $2 n m+1$ isolated vertices. Denote by $K_{m, 2 n m+1}$ the complete bipartite graph obtained by connecting each vertex in $V_{m}$ with each vertex in $V_{2 n m+1}$. Setting $S=V_{m}$ in Theorem [2.8, we deduce that $K_{m, 2 n m+1}$ contains no $[1,2 n]$-factors, and thus no $H_{n}$-factors. Moreover,

$$
o\left(K_{m, 2 n m+1}-V_{m}\right)=2 n m+1<(2 n+\epsilon)\left|V_{m}\right| .
$$

This completes the proof.

\section{Sufficient conditions for the existence of an $H_{n}$ - factor}

In this section, we present some sufficient conditions for the existence of an $H_{n}$-factor. For any vertex $v$ of $G$, denote by $N_{G}(v)$ the set of neighbors of $v$.

Theorem 3.1 Let $G$ be a graph without odd components. If for any non-adjacent vertices $u$ and $v$,

$$
\left|N_{G}(u) \cup N_{G}(v)\right|>\max \left\{\frac{g-2}{2 n}-1, \frac{2 g-4}{4 n+1}, \frac{g-1}{2 n+1}, 4 n-3\right\},
$$

then $G$ contains an $H_{n}$-factor.

Proof. Suppose to the contrary that $G$ contains no $H_{n}$-factors. By Theorem 2.5 , there exists a nonempty subset $S \subset V(G)$ such that the subgraph $G-S$ has at least $2 n s+1$ odd components, say, $C_{1}, C_{2}, \ldots, C_{2 n s+1}$, with each $C_{i}$ has no $H_{n}$-factors, where $s=|S|$. Let $c_{i}=\left|V\left(C_{i}\right)\right|$. Suppose that

$$
1 \leq c_{1} \leq c_{2} \leq \cdots \leq c_{2 n s+1}
$$

It is clear that

$$
2 n s+1 \leq c_{1}+c_{2}+\cdots+c_{2 n s+1} \leq g-s .
$$

Therefore

$$
\begin{aligned}
& c_{1} \leq \frac{g-s}{2 n s+1}, \\
& c_{2} \leq \frac{g-s-c_{1}}{2 n s} .
\end{aligned}
$$

It follows that

$$
\begin{aligned}
c_{1}+c_{2} & \leq \frac{2(g-s)}{2 n s+1}, \\
c_{2} & \leq \frac{g-s-1}{2 n s} .
\end{aligned}
$$


Moreover, the inequality (3.3) implies that $s \leq s_{*}$ where

$$
s_{*}=\frac{g-1}{2 n+1} .
$$

Let $u \in V\left(C_{1}\right)$ and $v \in V\left(C_{2}\right)$. Then

$$
\left|N_{G}(u) \cup N_{G}(v)\right| \leq s+\left(c_{1}-1\right)+\left(c_{2}-1\right) .
$$

By (3.4), we find that $\left|N_{G}(u) \cup N_{G}(v)\right| \leq h(s)$ where

$$
h(s)=\frac{2(g-s)}{2 n s+1}+s-2 .
$$

Note that the second derivative $h^{\prime \prime}(s)>0$. If $s \geq 2$, then we have

$$
\left|N_{G}(u) \cup N_{G}(v)\right| \leq \max \left\{h(2), h\left(s_{*}\right)\right\}=\max \left\{\frac{2 g-4}{4 n+1}, \frac{g-1}{2 n+1}\right\},
$$

contradicting to the condition (3.1). Otherwise $s=1$. In this case, if $c_{2} \leq 2 n-1$, then $c_{1} \leq 2 n-1$ by (3.2). By (3.6), we have

$$
\left|N_{G}(u) \cup N_{G}(v)\right| \leq c_{1}+c_{2}-1 \leq 4 n-3,
$$

contradicting to (3.1). So $c_{2} \geq 2 n$. It is easy to verify that any complete graph $K_{m}$ with $m \geq 2 n$ has an $H_{n}$-factor. Since $C_{2}$ contains no $H_{n}$-factors, we deduce that $C_{2}$ is not complete. So there exist vertices $u^{\prime}$ and $v^{\prime}$ which are not adjacent in $C_{2}$. By (3.5), we have

$$
\left|N_{G}\left(u^{\prime}\right) \cup N_{G}\left(v^{\prime}\right)\right| \leq s+c_{2}-2 \leq \frac{g-2}{2 n}-1,
$$

contradicting to (3.1). This completes the proof.

Observe that when $g \geq 8 n^{2}+2 n+2$, one has

$$
\max \left\{\frac{g-2}{2 n}-1, \frac{2 g-4}{4 n+1}, \frac{g-1}{2 n+1}, 4 n-3\right\}=\frac{g-2}{2 n}-1 .
$$

This results in the following corollary immediately.

Corollary 3.2 Let $G$ be a graph without odd components. If $g \geq 8 n^{2}+2 n+2$, and for any non-adjacent vertices $u$ and $v$,

$$
\left|N_{G}(u) \cup N_{G}(v)\right|>\frac{g-2}{2 n}-1,
$$

then $G$ contains an $H_{n}$-factor. 
Now we give another sufficient condition for the existence of an $H_{n}$-factor of a graph. A graph $G$ is said to be $k$-connected if it is connected when fewer than $k$ vertices are removed from $G$. Let $u$ and $v$ be non-adjacent vertices of $G$. Denote by $G+u v$ the graph obtained by adding the edge $(u, v)$ to $G$.

Theorem 3.3 Let $G$ be a k-connected simple graph with $g$ even. Let $u$ and $v$ be nonadjacent vertices of $G$ such that

$$
\left|N_{G}(u) \cup N_{G}(v)\right| \geq g-2 n k .
$$

Then $G$ has an $H_{n}$-factor if and only if the graph $G+$ uv has an $H_{n}$-factor. Moreover, the lower bound $g-2 n k$ in (3.7) is best possible.

Proof. The necessity is obvious. We shall prove the sufficiency. Suppose to the contrary that $G$ has no $H_{n}$-factors. Since $G$ is a connected graph with $g$ even, we deduce that $G$ has no odd components. By Theorem 2.5, there exists a nonempty subset $S \subset V(G)$ such that

$$
o(G-S) \geq 2 n s+1
$$

where $s=|S|$. Since $G$ is $k$-connected, it is easy to see that $s \geq k$. Let $C_{1}, C_{2}, \ldots, C_{q}$ be the components of $G-S$, and $c_{i}=\left|V\left(C_{i}\right)\right|$. Then $q \geq 2 n+1$ by (3.8). By Theorem 2.5, we can suppose that $c_{i}$ is odd and $C_{i}$ has no $H_{n}$-factors for any $1 \leq i \leq 2 n s+1$.

Let $u$ and $v$ be non-adjacent vertices. We have three cases.

(i) $u$ and $v$ belong to the same component of $G-S$. In this case, we can suppose that $u, v \in C_{i}$ for some $1 \leq i \leq q$. By (3.8), we have

$$
\left|N_{G}(u) \cup N_{G}(v)\right| \leq s+\left(c_{i}-2\right)=g-\sum_{j \neq i} c_{j}-2 \leq g-2 n s-2 \leq g-2 n k-2 .
$$

(ii) $u$ and $v$ belong to distinct components of $G-S$. In this case, we can suppose that $u \in C_{i}$ and $v \in C_{j}$ where $1 \leq i<j \leq q$. By (3.8), we have

$$
\left|N_{G}(u) \cup N_{G}(v)\right| \leq s+c_{i}+c_{j}-2=g-\sum_{h \notin\{i, j\}} c_{h}-2 \leq g-2 n k-1 .
$$

(iii) One of $u$ and $v$ belongs to the set $S$. Let $F$ be an $H_{n}$-factor of $G+u v$. Let $m$ be the total degree of vertices of $S$ in $F$, i.e.,

$$
m=\sum_{v \in S} d_{F}(v)
$$

Since the component $C_{i}$ contains no $H_{n}$-factors, and the edge $(u, v)$ is not contained in $G-S$, there exists an edge of $F$ connecting one vertex in $C_{i}$ and another vertex in $S$. Therefore, each $C_{i}$ corresponds an edge with one end in $S$. It follows that $m \geq 2 n s+1$. On the other hand, each vertex in $S$ has degree at most $2 n$ in $F$. So $m \leq 2 n s$, a contradiction. This proves the sufficiency. 
Now we shall prove that the bound $g-2 n k$ is best possible. Let $k$ be an odd number, and

$$
G=K_{k}+(2 n k+1) K_{1}
$$

Then $g=2 n k+k+1$ is even. It is easy to check that $G$ is $k$-connected and

$$
\left|N_{G}(u) \cup N_{G}(v)\right|=g-2 n k-1
$$

for any non-adjacent vertices $u, v \in V(G)$. It suffices to show that $G+u v$ contains an $H_{n}$-factor while $G$ does not.

Denote by $u_{1}, u_{2}, \ldots, u_{k}$ the vertices of the subgraph $K_{k}$, and by $v_{1}, v_{2}, \ldots, v_{2 n k+1}$ the remaining vertices in $G$. Suppose to the contrary that $G$ has an $H_{n}$-factor $F$. Then the degree of each vertex $v_{i}$ is at least 1 in $F$. Note that the neighbor of $v_{i}$ must be some $u_{j}$. So there exists some $u_{j}$ of degree at least $2 n+1$ in $F$. It follows that the degree of $u_{j}$ does not belong to the set $H_{n}$, a contradiction. Hence $G$ has no $H_{n}$-factors.

Now we shall show that $G+u v$ contains an $H_{n}$-factor for any non-adjacent vertices $u$ and $v$. In fact, since each vertex $u_{i}$ is saturated, we can suppose without loss of generality that the non-adjacent vertex pair $(u, v)$ is taken to be $\left(v_{2 n k}, v_{2 n k+1}\right)$. A factor $F$ of $G+u v$ consists of the edge $u v$ and the following $k$ components:

$$
\begin{aligned}
& G\left[u_{1}, v_{1}, v_{2}, \ldots, v_{2 n}\right], \\
& G\left[u_{2}, v_{2 n+1}, v_{2 n+2}, \ldots, v_{4 n}\right], \\
& \quad \vdots \\
& G\left[u_{k}, v_{2 n(k-1)+1}, v_{2 n(k-1)+2}, \ldots, v_{2 n k-1}\right] .
\end{aligned}
$$

It is straightforward to verify that $F$ is an $H_{n}$-factor. This completes the proof.

Let $H$ be an allowed prescription. In [9, Lemma (3.5)], Lovász gave the following result describing the $H$-decomposition of a graph when a vertex in $A_{H}$ is removed.

Lemma 3.4 (Lovász) Let $(A, B, C, D)$ be the $H$-decomposition of $G$. Let $v$ be a vertex in $A$, and $\left(A^{\prime}, B^{\prime}, C^{\prime}, D^{\prime}\right)$ the $H$-decomposition of the subgraph $G-v$. Then

$$
A^{\prime}=A-v, \quad B^{\prime}=B, \quad C^{\prime}=C, \quad D^{\prime}=D .
$$

Theorem 3.5 Let $G$ be a graph without odd components. Then $G$ contains an $H_{n^{-}}$ factor if the subgraph $G-v$ contains an $H_{n}$-factor for all vertices $v$.

Proof. Suppose that $G$ has no $H_{n}$-factors. Let $(A, B, C, D)$ be the $H_{n}^{*}$-decomposition of $G$. From the proof of Theorem 2.5, we see that $B=\emptyset$ and

$$
2 n|A|<c(D)
$$


Moreover, every component of $G[D]$ is odd.

Assume that $A \neq \emptyset$. Let $v \in A$, and $\left(A^{\prime}, B^{\prime}, C^{\prime}, D^{\prime}\right)$ be the $H_{n}^{*}$-decomposition of $G-v$. By Lemma 2.3, we have

$$
c\left(D^{\prime}\right) \leq 2 n\left|A^{\prime}\right|
$$

By (3.9), (3.10) and Lemma 3.4, we deduce that

$$
2 n|A| \leq c(D)-1=c\left(D^{\prime}\right)-1 \leq 2 n\left|A^{\prime}\right|-1=2 n(|A|-1)-1,
$$

a contradiction. So $A=\emptyset$. By Lemma 2.2 , any component of $G[D]$ is an odd component of $G$. But $G$ has no odd components, a contradiction. This completes the proof.

\section{References}

[1] J. Akiyama and M. Kano, Factors and Factorizations of Graphs, Lecture Notes in Math., vol. 2031, Springer, 2011.

[2] A. Amahashi, On factors with all degree odd, Graphs Combin. 1 (1985), 111-114.

[3] G. Cornuéjols, General factors of graphs, J. Combin. Theory Ser. B 45 (1988), 185-198.

[4] Y. Cui and M. Kano, Some results on odd factors of graphs, J. Graph Theory 12 (1988), 327-333.

[5] M. Kano, G. Y. Katona, and J. Szabó, Elementary graphs with respect to $f$-parity factors, Graphs Combin. 25 (2009), 717-726.

[6] M. Kouider and P. D. Vestergaard, Connected factors in graphs-a survey, Graphs Combin. 21 (2005), 1-26.

[7] M. Las Vergnas, An extension of Tutte's 1-factor theorem, Discrete Math. 23 (1978), 241-255.

[8] L. Lovász, The factorization of graphs, in: Combinatorial Structures and Their Applications (Proc. Calgary Internat. Conf., Calgary, Alta., 1969), 243-246, Gordon and Breach, New York, 1970.

[9] L. Lovász, The factorization of graphs. II, Acta Math. Hungar. 23 (1972), 223-246.

[10] M. D. Plummer, Graph factors and factorization: 1985-2003: A survey, Discrete Math. 307 (2007), 791-821.

[11] J. Topp and P. D. Vestergaard, Odd factors of a graph, Graphs Combin. 9 (1993), 371-381.

[12] R. Q. Yu and G. Liu, Graph Factors and Matching Extensions, Springer, 2009. 